A Careful Biomedicine? Generalization and Abstraction in RCTs.

Marsha Rosengarten \& Martin Savransky

Goldsmiths, University of London

\begin{abstract}
This article takes up biomedical and public health concerns about the difficulty of generalizing or extrapolating measurements of efficacy produced by the method of the randomized control trial (RCT) to wider populations. While explanations for the difficulty may be deduced from social studies of science that reveal the contingent and situated nature of trial findings, new conceptual tools are required to allow for the practical value associated with the possibility of their extrapolation. We argue that Alfred North Whitehead's concept of 'abstraction' can provide an alternative appreciation of some key aspects of the processes of knowledge-production of RCTs to enable a recasting of the problem of generalization. By proposing that generalization depends on relevant abstractions, we direct attention to the situated forms of care that this calls for. After showing the conceptual difference that the process of abstraction makes for understanding and extrapolating the situated nature of a research finding, we offer an interpretation of possible forms of care at work in efforts to devise Ebola adaptive trials. The example is offered as one possible basis for a reformulation of the logic of generalization.
\end{abstract}

Key words: generalization, randomized control trials, Alfred North Whitehead, abstraction, Ebola. 


\section{A Careful Biomedicine? Generalization and Abstraction in RCTs}

\section{Introduction}

Across the numerous fields of health and medical inquiry and intervention in evidencebased medicine (EBM), the randomized control trial (RCT) is regarded as the method best designed to statistically establish the validity of an intervention and, therefore, to address the question of whether it is better than another or better than none, that is, the question of equipoise (Freedman, 1987). It is the capacity of the RCT to suggest causal relationships under specified optimal conditions that has gained it pride of place for producing evidence of 'efficacious' interventions. As Rothwell states: 'Randomised controlled trials (RCTs) and systematic reviews are the most reliable methods of determining the effects of treatment' (2005:82). In order to provide such reliability, Rothwell adds, RCTs 'must be internally valid (ie, design and conduct must keep to a minimum the possibility of bias)' (2005:82). Nonetheless, an extensive array of debate ensues about how to overcome the difficulty of extrapolating or generalizing RCT evidence to other situations and, in reference to social interventions, whether this can be achieved at all (Bonell, 2002; Kippax and Van de Ven, 1998).

Within the field of social studies of medicine, ethnographic studies have shown that the difficulty of generalization resides with the presumption that RCT's produce an objective measure of probable causality. Such studies draw attention to the manner in which RCT findings are contingent on the many variable conventions and processes that comprise the method: for example, modes of standardization such as guidelines and protocols, marketing and drug regulation processes, data collection and review techniques (Cambrosio, Keating, Schlich and Weisz, 2009; Michael and Rosengarten, 2013; Timmermans and Berg, 2003; Will and Moreira, 2010). Thus, by contrast to the premises of the RCT to be discussed below, research in social studies of medicine suggests that evidence is not achieved simply by the detection of an externalized objective process able to transcend the situations in which it is collected (Robinson and Norris, 2001). Rather, 'evidence' is the outcome of situated practices and processes that labour towards its realization. Hence, since all effects are situated, emergent through the specific relational dynamics involved in their accomplishment-whether this is due to the situation of the RCT, in policy-making, or elsewhere-nothing would immediately warrant that they could happen elsewhere. 


\section{A Careful Biomedicine? Generalization and Abstraction in RCTs}

On this basis, and bearing in mind the importance of the RCT to EBM, this article takes up concerns about the difficulty of generalizing or extrapolating measurements of efficacy produced by the method of the RCT to wider populations and asks: what underpins the claim of generalizable evidence? Further, what alternative mode might enable findings more relevant to the situated nature of biomedical and other health interventions? To address the latter question, we introduce Alfred North Whitehead's concept of 'abstraction' and Maria Puig de la Bellacasa's notion of 'care', followed by a discussion of Ebola biomedical adaptive trials. The latter have been selected for the manner in which they reflect an increasing interest in research adaptation across biomedicine and public health. In this vein, they enable us to show how an engagement with the RCT method can be turned to reformulate generalization as a precarious and unstable process always dependent on relevant, situated abstractions. As such, adaptive trials also enable us to extend our proposition for a 'careful biomedicine,' one responsive to what is emerging in public health research apart from the reliance on the RCT (see for example, Walker and Jacobs, 2002; Valters, Cummings and Nixon, 2016).

\section{The logic of generalization: isolated causal mechanisms and the problem of externalized contingencies}

Hinging as it is on the minimization of biases that may confound the evidencing of the probability of a causal principle, the notion of internal validity within the method of the RCT involves the presupposition that an intervention's causal ability can be isolated and contrasted against a predetermined statistically significant outcome (Howick, 2011). By implication, this view of causality presupposes what sociologist of science Bruno Latour (1988) called a "process of purification", which selectively establishes distinctions between what counts as relevant data and what does not. Most notably, the conventional RCT method involves the randomization of human subjects, presumed to be equivalent as a 'population' within the trial, to either a 'control' one or more 'treatment' groups. Randomization is believed to eliminate 'bias,' such that calculations of statistical probability of an effect in the 'treatment' group/s can be contrasted with the 'control' for the purpose of generalizing the intervention's effect. Probability thus enables the 


\section{A Careful Biomedicine? Generalization and Abstraction in RCTs}

induction of causality without, in fact, explaining 'why something should or should not work’ (Timmermans and Berg, 2003:3).

Contrasted with what is understood as 'efficacy' testing by the RCT, 'effectiveness' is the degree to which the results can be found in a new situation and, for the most part, determined through observation. When effectiveness does not take place in the new situation, the reasons for this have traditionally been attributed to impeding external contingencies such as: less specialized skill levels of administration of the intervention than in that of the RCT; more challenging access and take up modes than in the RCT (Singal, Higgins, and Waljee, 2014); patients having co-interfering conditions than those in the RCT (Zwarenstein and Treweek, 2009); and, seen from a health programming perspective, that the circumstances for intervention 'change in rapid, complex and unpredictable ways' (Valters, Cummings and Nixon, 2016).

Across the different disciplinary fields seeking to redress what is viewed as a problem of translation from efficacy to effectiveness, while relying on the status of RCT for EBM, it is possible to identify an array of different strategy proposals. Amongst epidemiologists focused on translation to a clinical setting, there are calls for a continuum of trials, beginning with an explanatory mode associated with the conventional RCT efficacy testing, and followed by what are termed "pragmatic trials" allowing, in their design, for consideration of multiple so-called potential external contingencies alongside or post RCT efficacy testing (Singal, Higgins and Waljee, 2014:3; Treweek and Zwarenstein, 2009; Thorpe et al., 2009). Rod et al. (2014), for their part, argue for a need in public health intervention to move beyond the question of "what works", in favour of a more nuanced attention to what they call "social effectiveness" of intervention programmesnamely, an attention to the creation of shared understandings amongst researchers and practitioners, and the ways in which such intervention programmes reconfigure social relationships. Others, located within the primary health care and health programme implementation, propose a more participatory approach that involves practitioners and patients, or researchers and consumers, being able to exercise discrimination in the application of efficacy evidence and, in doing so, give consideration to other forms of knowledge (Greenhalgh, Howick and Maskrey, 2014; Walker and Jacobs, 2002; White, 2001). 


\section{A Careful Biomedicine? Generalization and Abstraction in RCTs}

Despite the somewhat different approaches for overcoming the difficulty of generalization set out above, what they share is the need for a compromise between an ideal of generalization and the situated nature of effects as made evident by social studies of medicine. It is this very notion of compromise that arguably also animates the distinction between 'efficacy' and 'effectiveness' findings. Whereas efficacy is assumed to be an achievement of RCT 'optimal' conditions for testing and thus likely to provide the most rigorous scientific evidence, effectiveness is understood as a situated challenge, susceptible to difficulties posed by the contingencies of each situation, and thus holding weaker epistemic and scientific potential.

Alongside these approaches, the practice of 'adaptation' is acquiring increasing interest across both medicine and public health for how it may enable more relevant evidence and, where possible, reduce the time it takes to achieve a desired result (Brown et al., 2009; Chow and Chang, 2008; Victora, Habicht and Bryce, 2004). To be sure, the array of adaptive types across these two overlapping fields is complex and it is beyond the scope of this article to enter into the 'whys' and 'wherefores' for their various differences. Here it will suffice to say that we are interested in approaches that involve 'adaptation' in situ. That is, where data is not only accumulated during the trial but is fed back into shaping aspects of the research in process (Brown et al., 2009). The reasons for our specific focus on adaptation in situ resonate in some crucial ways with the ongoing learning processes proposed by Valters, Cummings and Nixon (2016) in health implementation and, noted by Montgomery (2017:234), as a central feature of 'adaptive' biomedical trials.

Yet, despite the practical departure of 'adaptive' trial designs from pre-set 'optimal' conditions, by incorporating into the process of learning, data generated during the life of the trial, the presupposition of an isolated agent open for extrapolation to a new situation still forms part of their context of justification. In a comprehensive review of the pros and cons of adaptive trials versus the conventional RCT, Brown et al. (2009) acknowledge the rigidity of the latter method but nevertheless give expression to a prevailing view that the model of the adaptive trial is unable to provide answers to questions about probability for the purpose of generalization. As such, this presupposition is seen to weaken the epistemic importance of adaptive trials for EBM and public health policy. As 


\section{A Careful Biomedicine? Generalization and Abstraction in RCTs}

Montgomery (2017:246) notes: 'The more decisions or adaptations that are envisaged, the more difficult it is to know how the design will perform under different scenarios.'

Here we challenge the aforementioned devaluation of adaptive trials, and do so by problematizing the very presupposition of an isolated agent that underpins it. Labouring towards the idea of a 'careful biomedicine', we provide a cautious revaluation of adaptive trials that is appreciative of how the situated nature of an effect alters current assumptions about generalization. To do so, we first need to address what we have sought to highlight as a persistent paradox in the logic of the RCT and EBM, whereby contingencies are simultaneously acknowledged as central to the effects of an actual intervention, and eschewed from its own criteria of explanatory validity. Thus, in what follows we propose a different conceptual approach. An approach that is able to discern between the pragmatic value of the possibility of extrapolation apparent in the shift to adaptive trails, and the otherwise problematic consequences of the logic of generalization currently employed in EBM. In order to attempt this, we turn to the work of philosopher Alfred North Whitehead, his historical account of the development of modern mechanistic science, and his concept of 'abstraction.'

\section{The concept of abstraction and the history of science}

In his heterodox history of modern science, Whitehead (1967) traces the development of a mechanistic logic of causality with its concept of an ideally isolated system to Galileo. By setting up a purified experimental system that could single out a number of critical variables, Galileo was able to assert that the measure of speed produced within such a system was relevant to the behaviour of motion of physical bodies. According to Whitehead, the achievement of the isolated system, which involved the technical construction of an experimental apparatus able to exclude other factors, was a major technical accomplishment in abstraction, that is, the achievement of a partial taking from a whole. It is important to stress that, for Whitehead, abstraction is not just an elective mental operation but designates a process that is as crucial as it is ubiquitous, performed by all organisms. As such, it can neither be avoided nor overcome. Abstraction takes place at all levels of existence: a plant extracts water and minerals from the soil in which it situated and so, too, do thoughts extract from their situations. Indeed, when 'it abstracts, 


\section{A Careful Biomedicine? Generalization and Abstraction in RCTs}

thought is [...] exhibiting itself as an element in nature' (Whitehead, 1927:26), and creatively so. This process, moreover, is fundamental, such that 'we cannot think without abstractions' (Whitehead, 1967:73). But, while ubiquitous across nature, the process of abstraction is itself always situated, concerning each and every situation that different organisms help compose in their own specific ways: '[abstraction] occurs in a specific field or with regard to a specific problem; this is what provides it with both its purpose and its effectivity' (Halewood, 2011:6).

The Galilean event was an achievement of abstraction in the sense that Galileo succeeded in setting up a system of measurement and testing whose limited variables could be functionally isolated from the wider set of relationships that made up its environment, thus giving way to the development of experimental methodologies across the modern sciences. The effects of this event upon scientific thought have been far-reaching. Taking up the way in which physics has, since the Galilean event, come to predominate and model much of the thinking of the sciences, Whitehead expressed the concern -one that we are seeking to make resonate with the methods of the RCT and its evidence for generalization- that this event has since become the basis for a scientific materialism according to which nature is understood 'in terms of stuff, of matter [...] which has the property of simple location in space and time' (Whitehead, 1967:49). In other words, Galileo's achievement of a functionally isolated system was turned into an entire scientific worldview of ontologically isolated things, with the effect of becoming one of 'the most natural ideas for the human mind [such that] without these ways of thinking we [moderns] could not get our ideas straight for daily use' (Whitehead, 1967:52). What was the achievement of a specific experiment in physics, has acquired the status of a doctrine, of an 'orthodox creed' (Whitehead, 1967:51), and is now the cardinal methodological aim of efficacy testing in RCTs: the doctrine of simple location. According to the doctrine of simple location, nature is composed of isolated bits of matter 'that can be said to be here in space and here in time, or here in space-time, in a perfectly definite sense which does not require for its explanation any reference to other regions of space-time' (Whitehead, 1967:49).

Despite its ubiquitous presence in modern scientific thinking, one of the problems with this doctrine -as noted above in reference to the efficacy/effectiveness distinction- is that 


\section{A Careful Biomedicine? Generalization and Abstraction in RCTs}

to suppose that things exist in separation from each other makes it difficult to account for 'how one such substance can form a component in the make-up of another such substance' (Whitehead, 1927:26), that is, how one thing can be present in, and affect, another. For Whitehead, there is therefore a fundamental paradox at the heart of a science that espouses the assumption of simple location and employs a logic of induction to infer relations of causality, because

if in the location of configurations of matter throughout a stretch of time there is no inherent reference to any other times, past or future, it immediately follows that nature within any period does not refer to nature at any other period. Accordingly, induction is not based on anything which can be observed as inherent in nature. (Whitehead, 1967:51)

As a consequence of this history of scientific thinking, abstractions, which emerge out of the situated deployment of a 'method of suppressing what appear to be irrelevant details,' come to be deemed more real than -and thus replace- the complex concreteness of the situation from which it was abstracted. In this way, what was once discarded as irrelevant becomes simply invisible. As Whitehead puts it (1967:50), when an abstraction is confused with the concrete realities from which it draws, '[t]here is an error $[\ldots]$ of mistaking the abstract for the concrete. It is an example of what I will call the "Fallacy of Misplaced Concreteness".' There is an error because, as intimated above, those forms of knowledge that postulate clear and distinct things and qualities as simply located objects can only do so by enforcing upon every situation they study their own judgment of relevance, implicit or explicit in their own modes of abstraction. It is the abstraction that allows them to suppress what they consider to be irrelevant detail. Nothing guarantees, however, that what certain modes of abstraction consider relevant is indeed relevant to producing knowledge of, or intervening in, the situation from which they are abstracting (Savransky, 2016).

We can now see how biomedical and public health engagement with the question of generalization of RCT evidence stems from the event of an abstraction taken for actual, ontological, fact. And to the extent that this presupposes the doctrine of simple location not as an abstraction but as a fact of nature, it undermines the potential of the varying 


\section{A Careful Biomedicine? Generalization and Abstraction in RCTs}

attempts outlined above to rethink the problematic of generalization. Our aim, however, is not to denounce efforts to enable generalizations. What we suggest, by contrast, is that Whitehead's treatment of the concept of abstraction does more than offer a diagnosis of the history of biomedical culture. It provides an alternative way of evaluating, and of responding to, what is expressed in such attempts.

It follows from our excursus into Whitehead's concept of abstraction that, while all forms of knowledge create abstractions that simplify the complexity of concrete fact, no method of knowledge-production can constitute a 'gold-standard' as now presumed of the method of the RCT (Bonell, 2002; Timmermans and Berg, 2003). That is to say, no method of inquiry can, as a matter of principle, produce abstractions that are relevant to all situations. But this does not preclude the possibility that an abstraction may be relevant to many situations. As Whitehead (1967: 50) humorously acknowledged of those who turned the abstraction of a functionally isolated system into an ontological doctrine of simple location, 'the creed justified itself by the pragmatic test. It worked.' But, as many public health practitioners working to implement an intervention will be aware, the adjustment of any efficacy measures to so-called external contingencies always involves a situated test. It may work, it may even work frequently, but only the doctrine of simple location warrants that it should always work as a matter of principle. Put differently, abstractions are situated in the sense that they emerge as a response to a specific problem. They can indeed prove to be relevant in many situations. But to abstract is not to generalize. To the extent that the notion of generalization stems from the abstraction of an isolated system, Isabelle Stengers (2008:96) rightly remarks that abstractions, for Whitehead, 'are not generalizations.' Whereas generalizations are always concerned with the problem of representation, and their aim is that of issuing probabilistically accurate, objective, or true statements of an independent world, the function of abstractions is 'not to produce new definitions of what we consensually perceive and name, but to induce empirically felt variations in the way our experience matters' (Stengers 2008: 96).

Indeed, abstractions can, but need not be, statements at all, and they are not governed by the requirements of representation. The example of the abstracting plant mentioned above is illustrative: a plant abstracts water and minerals from the soil, but of course, it needs not produce a representation of them in order to experience the variations that these 


\section{A Careful Biomedicine? Generalization and Abstraction in RCTs}

abstracted elements induce in its experience. At the human end of the spectrum, Stengers (2008:96) illustrates this feature of abstraction by attending to the way in which the abstraction of a circle operates in mathematical thought. As far as mathematics is concerned, the circle need not refer to any actual circle. The function of the circle, moreover, is not to instruct mathematicians on the common properties of all actual circles -were such a thing to exist- but to 'lure mathematicians into adventures which produce new aspects of what it means to be a circle into a mathematical mode of existence.'

The task of abstractions, therefore, is not to be 'true', but to be relevant (Savransky, 2016). Their aim is to 'act as "lures", luring attention towards "something that matters", vectorising concrete experience' (Stengers, 2008: 95). Because their task is to be relevant to the concrete situations in which they operate, the relevant scope of an abstraction can never be guaranteed. Despite the premise of EBM, according to which the production of 'best evidence' hinges on the particular method used to collect it, to attend to the processes of abstraction undertaken by method-driven research is to trouble expectations of the replication of results in a different situation, particularly the expectation that such an event of replication can be determined methodologically, as a matter of principle. Instead, relating to abstractions is always a pragmatic challenge, a question of the relevance of a specific abstraction to a particular situation. This pragmatic challenge, we argue, demands that one learns to care for the ways in which abstractions operate in concrete situations.

\section{Caring for Abstractions}

To speak of a care of abstractions involves, as Maria Puig de la Bellacasa (2011:100) proposes, a 'transformative ethos' that introduces the question of "how to care" in each situation'. But here the notion of care does not evoke the connotations of a form of warm, pleasant affection, and it cannot be reduced to the clinical side of a medical practice -or other practices involved in the implementation of EBM- that would have rigorous scientific knowledge as their counterpart. In Puig de la Bellacasa's ethico-political rendering of the term, care is an attentive response to the systematic neglect and undervaluing of some issues at the expense of others. Indeed, for Puig de la Bellacasa (2017: 15) care thus involves an entire 'politics of knowledge at the heart of technoscientific, naturecultural world.' 


\section{A Careful Biomedicine? Generalization and Abstraction in RCTs}

The notion of 'care' is thus a particularly apposite notion to explore the uses of abstractions: to interrogate what forms of neglect they involve, and the extent to which their criteria of relevance are adequate to the situations in which they operate. It is not only a matter of detecting what is and is not included but also about caring for, as Puig de la Bellacasa (2011: 96) states: 'what this thing could become' within current constraints. To care for abstractions is thus to become attentive to, and vigilant of, the particular modes of abstraction and the kinds of dynamic connections and disconnections that may be possible to cultivate with the situation of concern. In this sense, the notion of care does not simply provide an analytical framework through which to interrogate techno-scientific practices from without, but enables a different sort of interrogation, more generative and generous, with which the practices of biomedicine may be directly concerned. This is the question of how a certain research methodology may continously adjust its modes of abstraction and thus seek to become more careful.

In the next section we attend to the efforts within biomedicine that, by their design, displace the efficacy and effectiveness distinction of the RCT. We suggest that a nascent orientation to the use of abstractions and away from the concern with 'contingencies', posited as external by prevailing biomedical and public health thinking, can be appraised in the practice of 'adaptive biomedical trials' despite their purported adherence to a mode of causality that raises concern about generalization as a logical consequence of its methodology (Brown et al., 2009).

\section{Abstraction in Adaptive RCTs}

In what follows, our aim is to constructively contribute a different understanding of the nature of evidence and what it means to extrapolate a situated effect when caring for abstractions. This involves dramatizing a practice, albeit unacknowledged by biomedicine, of caring for abstractions as illustrated in the shift to 'adaptive trials', namely, clinical trials which, in different ways, modify some of their parameters and protocols in view of their ongoing outcomes. To do so, we draw on the constructive characterisation by statistician Steve Kanters (2014) as fruitful responses to the evolving demands of the Ebola outbreak during 2014 - 2016. It is the combination of the successful development 


\section{A Careful Biomedicine? Generalization and Abstraction in RCTs}

of an alternative mode of abstraction evinced in one Ebola vaccine trial, together with an emerging revaluation of its evidence-value in the discourse of some statisticians, which initially enabled us to grasp adaptive trials' practical value for reformulating the efficacy/effectiveness distinction now underpinning the notion of 'best evidence'. To be sure, there are many other adaptive approaches that we might have selected- notably, those concerned with non-biomedical interventions (see for example, Brown et al., 2009; Victora, Habicht and Bryce, 2004). But given that it is a certain logic of scientific evidence which gives weight to the RCT method, and that central to our proposition for a careful biomedicine is to rethink the presuppositions of generalization, we believe a conceptually stronger case for our proposition may be achieved by focusing on adaptation in biomedicine. That said, it is important to note that our discussion below is not intended as a comprehensive examination of the socio-technical complexities of the adaptive trial, and it does not seek to mobilize the cases we discuss to make evidential appeals of its own. Such an aim, although urgent and important, is beyond our current scope.

In what follows, we pursue a speculative and more modest endeavour, of seeking to disclose, in some of the methodological and evaluative dimensions of three adaptive trials, gestures that may be generative of a more careful way of engaging the situated processes of abstraction and generalization involved. Indeed, our efforts are themselves abstractive in their own way, devised to vectorize the possibility of a different mode of engagement with biomedical evidence in public health, one whose full realization will no doubt require further, in-depth, situated attention to the complexities of each trial in question.

In his endorsing statements, Kanters provides only a sketch of two adaptive trials: (1) TKM-Ebola later not found to reach its target effect (Dunning et al., 2016), and (2) Zmapp, halted due to the constraints of what emerged as a declining epidemic (The Prevail Writing Group, 2016). Nevertheless, his argument is noteworthy, for it is crucially concerned with aligning the need to 'acquire critical scientific knowledge as well as help patients in dire need of treatment.' The need to do so, framed according to bioethics, was expressed in response to the view that the conventional RCT is the most ethical mode of research as it involves pre-safety testing and, as we have noted above, it is also regarded as the most epistemically robust method to achieve generalizable claims due to the role of randomization in statistical comparison. However, for Kanters and many others reviewing 


\section{A Careful Biomedicine? Generalization and Abstraction in RCTs}

the various adaptive trials proposed and undertaken during the epidemic, the strict nature of the conventional RCT is precisely its limitation (see Whitehead et al., 2016; Yin, Lam, and Shi, 2017). As Kanters (2014) states, the conventional RCT relies on 'pre-planned and assumed knowledge' which limits what can be learnt and achieved during its undertaking- a concern made most pertinent in response to the urgency of Ebola. Emphasizing what is most problematic about the conventional RCT when seeking a novel intervention, he goes on to say:

In reality ... many trial designs would be altered if much of the knowledge accrued over the course of the trial were known beforehand. By the time we realise that trials should have been changed, or adapted, the strict rules of standard clinical trials often do not permit protocol change.

Hence, Kanters suggests, adaptation was required not because it would generally provide a better RCT design or more objective and generalizable measures, but above all, because of the need to produce knowledge and biomedical technologies in a situation where ongoing learning was urgently required. His description of the process of adaptation emphasizes the monitoring of effects during the trial, and adapting it within the research undertaking, that is, in-situ. In place of imposing on its test-population a criterion of relevance fully determined in advance, hypothesizing a causal mechanism and methodologically establishing whether or not it has a probabilistic beneficial or harmful effect to determine the trial result, the TKM-Ebola and Zmapp trial designs meant that their results would be contingent on relevant, ongoing adjustments to the demands of the situation. Because of the potential risk of adverse reactions to a novel intervention, the TKM intervention was introduced into the bloodstream over a minimum two-hour period, during which clinical monitoring for reactions took place. Moreover, given the constraints on providing care in a complex, poorly equipped setting of the West African epidemic, the trial's lead physician decided on a daily basis how many new participants could be included (Dunning et al., 2016). By contrast, the ZMapp trial was designed to test a complex set of interventions and used a process of randomization that involved a response-adaptation mode, shifting patients to a treatment arm as the trail progressed. It also included close monitoring of a dosing pattern in-situ (Kirchoff and Pierson, 2017). 


\section{A Careful Biomedicine? Generalization and Abstraction in RCTs}

While on paper the trials did not ostensibly depart from seeking to identify a causal mechanism, they (like other adaptive trials) nevertheless could be said to have developed a mode of abstraction that was more open, and more responsive, to actively look for data that could lure biomedical practices to 'something that matters.' In short, the rapidly shifting and expanding nature of the situation was prioritized over the usual requirements of the methodological gold-standard, with a view to taking care to abstract from the situation in ways that would be relevant and responsive to its evolving and rapidly changing character. Indeed, the situation shifted so rapidly that, although both trials did generate positive results, neither were able to achieve a statistically significant result before discontinuing in the face of the decline of the epidemic.

Writing without the knowledge of the outcomes of the two trials, Kanters (2014) expressed the following qualification and justification for his endorsement of their design:

The upcoming Ebola trials will be marred with unknowns that are required to conduct standard trials. These include uncertainties about the interventions, such as efficacy, dosage, co-interventions, and adverse events, and uncertainties about the population as the epidemic continues to cause societal and economic turmoil.

To be sure, as we noted above, a version of isolationism pertaining to the mechanistic logic of causality remained present in the above cited Ebola adaptive trials, such that the intervention was still conceived as the causal feature of the sought effect. But unlike the conventional/non-adaptive RCT, this was a functional isolation of a causal factor, devised and continuously adjusted in-situ for the purposes of discerning something that matters. In other words, the question of relevance, of what does indeed matter in a particular situation, was not completely determined by method but retained a degree of openness. Indeed, it became part of the very research question, allowing for a careful responsiveness to the situation in the ongoing development of the trial's abstractions.

To be sure, this does not preclude successful adaptive trial findings being taken to refer to a generalizable simple location. Nothing in Kanter's proposal for adaptation or, adaptive trials more generally, prevents the findings from later becoming subject to the logic that what works here will work somewhere else. But this should not dissuade us from 


\section{A Careful Biomedicine? Generalization and Abstraction in RCTs}

entertaining the shift to adaptation as a vector for extending our thinking on the possibilities afforded by the conventional RCT. Despite other conceptual limitations and socio-technical complexities, we suggest that the very shift to adaptation in-situ in the face of a public health emergency makes the importance of taking care of our abstractions resonate.

We turn now to 'Ebola ça suffit!', a trial conducted in Guinea to produce a vaccine against Ebola and described by its sponsor, the World Health Organisation, as using a phased-rollout RCT method (Pulliam, Bellan, Gambhir, Meyers and Dushoff, 2015). In brief, a laboratory developed vaccine was put to the test through a ring design tailored to respond to what had become a diminishing number of Ebola cases as the pattern of the epidemic, itself, shifted to small outbreaks: when Ebola flared up in a village, researchers vaccinated all the contacts of the sick person who were willing - the family, friends and neighbours - as well as their immediate contacts. The cluster outbreaks were assigned to either receive the vaccine immediately or three weeks after Ebola was confirmed. The process of assignment was not done according to the usual process of randomization, but by prioritizing vaccination of clusters experiencing high infection and, according to Pulliam et al. (2015:1134), conferring 'the highest likelihood of benefit to those at highest risk, thereby reducing the total risk to trial participants relative to a non-risk prioritized design.' Among the 2,014 people vaccinated immediately, there were no cases of Ebola from 10 days after vaccination - allowing time for immunity to develop and providing a markedly different pattern to that observed in situations where no such intervention had been possible. There were 16 cases of Ebola infection out of 2,380 in the clusters with delayed vaccination (Krause, 2015).

No doubt, some would claim that the success of 'Ebola ça suffit!' can be explained as due to the particular nature of the intervention, that is, its situated requirement involving one injection to the arm tested in-situ in villages rather than a more complex intervention and varying set of circumstances. And, as is the case with any intervention, the long-term effects of the vaccine remain unknown. Notably, its duration of protection is yet to be observed. But if we entertain the possibility that the research design and intervention may have so far proven relevant, we may appreciate how the usual contrast between RCT control (efficacy) and real world (effectiveness) conditions loses its hold. In this 


\section{A Careful Biomedicine? Generalization and Abstraction in RCTs}

particular case, it was replaced by a staged delivery process to vectorize an abstraction in order to alter an ongoing course of deadly events of infection through exposure to the body fluids of an infected individual (Beeching, Fenech, and Houlihan, 2014).

In attending to the use of abstractions, it is necessary to remember that our discussion of the successful Ebola trial should not be taken to suggest that the staged design is necessarily appropriate to other situations. Unlike the dominant logic of generalization, a pre-fixed form of caring for abstractions can never become crystalized in the protocols of a general methodology. Hence, it is of import here because it was the situation itself, the interaction between the trial and the population with which it was concerned, that was relied on to achieve the possibility of a successful vaccine. This certainly poses a significant contrast to the conventional notion of 'efficacy' which, with its emphasis on 'internal validity' as an achievement of pre-fixed controlled conditions, suggests that for an effect to be proven efficacious it would need to obtain in spite of the situation that is concerned with its success. By contrast, what we are seeking to make visible is that the success of the adaptive 'Ebola ça suffit!' trial depended on the careful crafting of a relevant abstraction, where a partial knowledge of the processes of viral, body and vaccine was synthesized to achieve a concrete effect.

While the RCT provides a method for generalizable knowledge -or would claim to do so in principle-caring for abstractions demands that one learns how to come to know a situation depending on what the latter itself demands. Thus, it can be argued that shifts toward adaptation offer an empirical gesture beyond that of the prevailing logic of generalization. Although adaptation of the RCT is not new (Montgomery, 2017), what we have sought to disclose is a way of interpreting the differences that adaptive trials might make, and the potentialities they may yield. The adaptive mode gestures towards a commitment to take care of how an abstraction may operate in relation to those with whom it is tested, and in relation to those that put it to the test, without presuming to fully know in advance how this should come about.

As such, although it inevitably proceeds from a pre-determined protocol for adaptation, the protocol itself does not entirely dictate what would count as relevant and what could be discarded as irrelevant detail. Instead it gives recognition to the need for a careful 


\section{A Careful Biomedicine? Generalization and Abstraction in RCTs}

attention to and discernment of what becomes relevant in the course of its own unfolding. Doubtless, as Montgomery (2017:236) has argued, adaptive trials still involve forms of neglect of the radically unpredictable. But it is also apparent to us that they make the idea of a careful biomedicine possible: a kind of biomedical research for which no contradiction between rigorous scientific evidence and situated relevance exists, a possible biomedicine for which 'the best' evidence for medical practice and public health policy is evidence produced with care. In sum, adaptation is conceived in a manner that offered the possibility for the tested intervention to become efficacious at the same time as it became effective (on similar designs for other conditions see, for example, Chow and Chang, 2008).

\section{Conclusion}

The work of abstraction suggests that stabilities - the achievement of an effect in and across bodies for a period of time as may be observed in EBM-are always partial and dynamic achievements rather than mere givens. By adopting this view, there is the possibility of associating with the question of biomedical and other health interventions a form of inquiry that would neither dismiss outright the possibility that abstractions can work in many situations, nor attempt to abstract a claim to efficacy at all costs. This is what we have tentatively called a careful biomedicine: one for which 'the best' evidence for medical practice and public health policy is evidence produced with care.

Caring for abstractions involves taking into account that the challenge of relevance that research abstractions face can never be dispelled once and for all, by the sort of methodological principles exemplified by the RCT with its notion of 'optimal conditions.' When research and policy is underpinned by a presumption of isolated causality, it is thus not sufficient to presume that the problem of generalization resides elsewhere with externalized contingencies. To put this a little differently, if we can never be sure to include all the elements of a situation, it is only by attending to the work of abstraction in the achievement of an effect that a careful biomedicine and health intervention become possible. Imagining a careful biomedicine, we suggest, allows for the possibility of an intervention attuned to the situated nature of its claims, while guarding against the fallacy 


\section{A Careful Biomedicine? Generalization and Abstraction in RCTs}

of presupposing that the abstraction of efficacy can, in and of itself, become an effective generalization.

In this way, the idea of a "careful biomedicine" perhaps provides an opportunity for reimagining a different sort of relation between the biomedical sciences and critical public health research and intervention. Rather than stop at a general critique of RCTs by highlighting the often problematic nature of its evidential claims, our proposition of a careful biomedicine suggests that the critical health field, with its persistent practices of learning to pay attention to what biomedical modes of abstraction neglect, has crucial lessons to offer to biomedicine itself. Ones that not only emphasize the latter's limitations but can also proffer alternative propositions for its transformation. As such, it turns the tables, so to speak, on the manner in which biomedical evidence is evaluated. Rather than depend on the internal validity of the methodological rigour of the RCT, the notion of a careful biomedicine places relevance as the situated test of any intervention, and it emphasizes that relevance cannot be achieved without an ongoing process of learning, on each occasion, how to craft abstractions that may induce felt variations in the way our experience matters.

\section{References:}

Beeching, N. J., Fenech, M. and Houlihan, C.F (2014) Ebola Virus Disease, The BMJ 349: g7348.

Bonell, C. (2002) The Utility of randomized controlled trials of social interventions: An examination of two trials of HIV prevention, Critical Public Health 12:4, 321334.

Brown, C.H., Ten Have, T.R., Jo, B., Dagne, G., Wyman, P.A., Muthén, B. and Gibbons, R.D. (2009) Adaptive Designs for Randomized Trials in Public Health Annu Rev Public Health. 29 (30): 1-25.

Cambrosio, A., Keating, P., Schlich, T. and Weisz, G. (2009) Biomedical Conventions and Regulatory Objectivity: A Few Introductory Remarks, Social Studies of Science 39 (5): 651-664.

Chow, S. and Chang, M. (2008) Adaptive design methods in clinical trials-a review, Orphanet Journal of Rare Diseases 3:11. 


\section{A Careful Biomedicine? Generalization and Abstraction in RCTs}

Dunning, J., Sahr, F., Rojeck, A., Gannon, F., Carson, G., Idriss, B., et al. (2016) Experimental Treatment of Ebola Virus Disease with TKM-130803: A SingleArm Phase 2 Clinical Trial. PLoS Med 13(4): e1001997.doi:10.1371/journal.pmed.1001997

Freedman, B. (1987) Equipoise and the Ethics of Clinical Research, The New England Journal of Medicine, 317: 141-5.

Greenhalgh, T., Howick, J. \& Maskrey, N. (2014) Evidence Based Medicine: A Movement in Crisis?, BMJ 348, no. jun13: g3725-g3725, doi:10.1136/bmj.g3725.

Halewood, M. (2011) A.N. Whitehead and Social Theory: Tracing a Culture of Thought (London and New York: Anthem Press).

Howick J. (2011) The Philosophy of Evidence-Based Medicine (Oxford, UK: WileyBlackwell).

Kanters, S. (2014) Evaluating Ebola interventions: adaptive designs should be commonplace, The Lancet Global Health Blog 10 October. Retrieved from http://globalhealth.thelancet.com/2014/10/10/evaluating-ebola-interventionsadaptive-designs-should-be-commonplace [November 2014].

Kippax, S. and Van den Ven, P. (1998) An epidemic of orthodoxy? Design and methodology in the evaluation of the effectiveness of HIV health promotion. Critical Public Health 8(4): 371-386.

Krause, P.R. (2015) Interim Results from a Phase 3 Ebola Vaccine Study in Guinea, The Lancet 386 (9996), pp. 831-33.

Kirchoff, M.C. and Pierson, J.F. (2017) Considerations for Use of Investigational Drugs in Public Health Emergencies Therapeutic Innovation \& Regulatory Science 51 (2) $146-152$.

Latour, B. (1988) Science in Action: How to Follow Scientists and Engineers Through Society (Cambridge, Mass: Harvard University Press).

Michael, M. and Rosengarten, M. (2013) Innovation and Biomedicine: Ethics, Evidence and Expectation in HIV (London and New York: Palgrave MacMillan).

Montgomery, C. (2017) From Standardization to Adaptation: Clinical Trials and the Moral Economy of Anticipation_Science as Culture 26 (2): 232-254.

Montgomery, C. and Pool, R. (2017) From 'trial community' to 'experimental publics': how clinical research shapes public participation Critical Public Health 27, 1: 5062. 


\section{A Careful Biomedicine? Generalization and Abstraction in RCTs}

Puig de la Bellacasa, M. (2011) Matters of Care in Technoscience: Assembling neglected things, Social Studies of Science 41 (1), pp. 85-106.

Puig de la Bellacasa, M. (2017) Matters of Care. Minneapolis: University of Minnesota Press.

Pulliam, J.R.C., Bellan, S.E., Gambhir, M., Meyers, L.A. and Dushoff, J. (2015) Evaluating Ebola vaccine trials: insights from Simulation, The Lancet 15(10):1134.

Robinson, J.E. and Norris, N. F. J. (2001) Generalisation: the linchpin of evidencebased practice? Educational Action Research, 9:2, 303-310

Rod, M.H., Ingholt, L., Sørensen, B.B. and Tjørnhøj-Thomsen, T. (2014) The spirit of the intervention: reflections on social effectiveness in public health intervention research, Critical Public Health, 24:3, 296-307.

Rothwell P.M. (2005) External validity of randomised controlled trials: "to whom do the results of this trial apply?" The Lancet, 9453 (365), pp. 82-93.

Savransky, M. (2016) The Adventure of Relevance: An Ethics of Social Inquiry (Basingstoke and New York: Palgrave Macmillan).

Singal, A.M., Higgins, P.D.R. and Waljee A.K. (2014) A Primer on Effectiveness and Efficacy Trials Clinical/Narrative Review, Clinical and Translational Gastroenterology 5(1):e45.

Stengers, I (2008) A Constructivist Reading of Process and Reality, Theory, Culture and Society, 25, 4: 91-110.

The Prevail Writing Group (2016) A Randomized, Controlled Trial of ZMapp for Ebola

Virus Infection New England Journal of Medicine 375:1448-56.

Thorpe K.E., Zwarenstein, M, Oxman, A.D., Treweek, S., Furberg C.D., Altman D.G.

... Chalkidou, K. (2009) A pragmatic-explanatory continuum indicator summary (PRECIS): a tool to help trial designers, Journal of Clinical Epidemiology, 62(5):464-75.

Timmermans, S. and Berg, M. (2003) The Gold Standard: The Challenge of EvidenceBased Medicine and Standardization in Health Care (Philadelphia Temple University Press). 


\section{A Careful Biomedicine? Generalization and Abstraction in RCTs}

Treweek, S. and Zwarenstein, M. (2009) Making trials matter: pragmatic and explanatory trials and the problem of applicability Trials, 10:37 Retrieved http://www.trialsjournal.com/content/10/1/37 [14 October 2014]

Valters, C., Cummings, C. and Nixon, H. (2016) 'Putting learning at the centre Adaptive development programming in practice,' Report by Overseas Development Institute, London, United Kingdom.

Victora, C.G., Habicht, J. and Bryce, J. (2004) Evidence-Based Public Health: Moving Beyond Randomized Trials, American Journal of Public Health 94, (3):400-405.

Walker, C. and Jacobs, S. (2002) Social structures of science and approaches to outcomes-based medical research, Critical Public Health 12:4: 309-320.

Will, C.M. and Moreira, T. (2010) Medical Proofs, Social Experiments: Clinical Trials in Shifting Contexts (Farnham: Ashgate).

White D. G. (2001) Evaluating evidence and making judgements of study quality: Loss of evidence and risks to policy and practice decisions, Critical Public Health (11)1:3-17.

Whitehead, A. N. (1927) Symbolism: Its Meaning and Effect (New York: Fordham University Press).

Whitehead, A. N. (1967) Science and the Modern World (New York: Free Press).

Whitehead, J., Olliaro, P., Lang, T. and Horby, P. (2016) Trial design for evaluating novel treatments during an outbreak of an infectious disease Clinical Trials $13(1): 31-38$.

Yin, G., Lam, C.K. and Shi, H. (2017) Bayesian randomized clinical trials: From fixed to adaptive design Contemporary Clinical Trials 59:77-86.

Zwarenstein, M. and Treweek, S. (2009) What kind of randomised trials do patients and clinicians need? Annuals of Internal Medicine 150(10): JC5-3. 